\title{
Application of Spectral Unmixing on Hyperspectral Data of the Historic Volcanic Products of Mt. Etna (Italy) ${ }^{+}$
}

\author{
Vasiliki Daskalopoulou ${ }^{1,2, *}$, Olga Sykioti ${ }^{1}$, Catherine Karagiannopoulou ${ }^{1}$, \\ Konstantinos Koutroumbas ${ }^{1}$ and Athanasios Rontogiannis ${ }^{1}$ \\ 1 Institute for Astronomy, Astrophysics, Space Applications and Remote Sensing, National Observatory of \\ Athens, 15236 Athens, Greece; sykioti@noa.gr (O.S.); kat.karagiannopoulou@gmail.com (C.K.); \\ koutroum@noa.gr (K.K.); tronto@noa.gr (A.R.) \\ 2 Department of Physics, Faculty of Astrophysics and Space Physics, University of Crete, 70013 Herakleion, \\ Greece \\ * Correspondence: vdaskalop@noa.gr; Tel.: +30-210-810-9183 \\ + Presented at the 2nd International Electronic Conference on Remote Sensing, 22 March-5 April 2018; \\ Available online: https://sciforum.net/conference/ecrs-2.
}

Published: 22 March 2018

\begin{abstract}
Considering that ground areas with intense compositional variability appear as mixed pixels in hyperspectral data, we focus on the mixing problem imposed by the various volcanic products found in the vicinity of Mt. Etna's volcanic craters. Mt. Etna, which is one of the most active volcanoes globally, is a generator of diverse mineralogical environments. Therefore, the inherent abundant information of hyperspectral imagery of the volcanic edifice calls for the use of timeefficient and accurate spectral unmixing methods in order to unravel the data. Lava flows (LFs) and related products from the historical 1536-1669 era were selected based on their distinct spatial distribution and lava field segregation. Based on the selection of appropriate pixel representatives, distinct optimizing signal transformations were implemented, with the most dominant being the Fourier transform, in order to use the data in the linear least squares unmixing (LLSU) and bilinear unmixing (BLU) methods. We thus report the results of the historic lava flow characterization and respective abundance analysis qualitatively and quantitatively, evaluated through the structural similarity index (SSIM) of each method. Ultimately, method intercomparison provided the optimum selection for volcanic product segregation.
\end{abstract}

Keywords: hyperspectral data; Etna; lava flow characterization; linear spectral unmixing; bilinear spectral unmixing; fast Fourier transform; structural similarity index

\section{Introduction}

As part of the plethora of technologies exploited in remote sensing, hyperspectral imaging (HSI) sensors provide information on hundreds of distinct and contiguous channels of the electromagnetic spectrum, thus enabling the identification of multiple ground objects through their detailed spectral profiles. However, restrictions on the spatial resolution of HSI data, the multiple scattering of the incident light between objects, and microscopic material mixing together form the mixed pixel problem. Pixels are identified as mixed when they are composed of the spectral signatures of more than one ground object. Therefore, we adopted the linear and non-linear spectral mixing techniques $[1,2]$, which model the pixel spectra as a combination of pure components (endmembers) weighted by the fractions (abundances) that contribute to the total reflectance of the mixed pixel. Ideally, each selected endmember from the HSI under study has the maximum possible abundance of a single 
physical material present and minimum abundance for the rest of the physical materials. Spectral unmixing (SU) consists of two main substages: (a) endmember extraction; and (b) abundance estimation [3]. In addition, certain signal transformations are adopted prior to the performance of SU $[4,5]$. The determination of the number of endmembers is critical, since underestimation may result in a poor representation of the mixed HSI pixels, whereas overestimation may result in an overly segregated area. Clearly, the ultimate success of unmixing depends heavily on the appropriate selection of the endmembers. In addition, since only a small number of the available materials' spectra are expected to be present in a single pixel, the abundance vectors are often sparse in nonzero entries.

In mineralogy, the identification of the mineral constituents of major rock types is typically approached using unmixing methods [1]. The Sicilian volcano, Mt. Etna, is an intriguingly diverse case study, since it is one of the most active stratovolcanoes globally. Its intense volcanic activity [6], geomorphological complexity [7] and well-documented flank eruptions [8] perplex the remote sensing monitoring of the bulk volcanic edifice. Various spectroscopy studies $[9,10]$ over the volcanic area have examined the mineralogical composition of the extensive lava fields. Nontheless, there are no findings concerning Etna's lava flow (LF) delineation through unmixing, which further intensifies the need for multitemporal analysis of the volcano.

In this paper, we focus on the accurate estimation of fractional abundances of the deposited volcanic products on older lava fields. For this purpose, a NASA Hyperion, on board the Earth Observing Mission 1, HSI image of Etna was selected and for the sub-pixel analysis we proposed four signal transformations that can be assimilated in linear least squares unmixing (LLSU). Our approach was narrowed to the part including Etnian historical eruptions on the sensor's field of view, namely between 1536-1669 AD, where deposited products present lower correlation coefficients than those of younger lava flows. The latest geological map of Etna was used as the ground truth [7]. The resulting abundances from each method were quantitatively evaluated via the structural similarity index (SSIM) [11] and, finally, a comparative analysis of the selected methods was carried out.

\section{Study Area and Methodology}

In this section, we define the key characteristics of Etna's morphology and describe the implemented methods for the linear and bilinear unmixing of the historic lava fields.

\subsection{Study Area}

Mt. Etna is a large basaltic complex stratovolcano with a broad base diameter of $1.178 \mathrm{Km}^{2}$. Its volcanism started approximately $500 \mathrm{ka}$ ago, from a submarine fissure of the Gela-Catania basin. It is characterized by two major components: (i) the central summit craters, and (ii) the flank eruptions. Summit events are consistent for many years, while flank eruptions occur every few years and are correlated with tectonic faults and shallow seismic activity [12]. Etna has the ability to change its land field rapidly, vigorously and continuously. It is capable of producing both brief paroxysmal episodes and long-standing, relatively milder eruptions. During the last 100 years Mt. Etna has produced on average $10^{7} \mathrm{~m}^{3}$ of new lava per year [8], both from its summit craters and from its flank areas. The youngest active bulks of Etna are Ellitico and Mongibello. We focus our research on the persistent activity of the Mongibello volcano, which entails the major volcanic formation of Torre del Filosofo. It contains voluminous products younger than $122 \mathrm{BC}$, with continuous temporal succession and similar lithologies. The formation is divided into three sub-formations formed in different time periods [7]:

- Mongibello Filosofo 1 (MF1): The first sub-formation with volcanic products from 122 BC-1669 AD. The effusive products originate from intense summit activity and are combined with intermediate flank eruptions. As expected, MF1 products follow the mountain morphology and spread radially from the main volcanic volume.

- Mongibello Filosofo 2 (MF2): Includes the time interval of the post-1669 AD eruption period to the pre-1971 AD eruption period. 
- Mongibello Filosofo 3 (MF3): Contains the most recent and well-documented summit/flank eruptions and flows eastward to the Valle del Bove depression .

MF1 is ideal for testing unmixing processes, since it contains mostly altered lavas with high reflectance. The respective lava fields are distinct, voluminous and often do not mingle with younger lavas. Considering the Hyperion's limited swath range and spatial resolution over Etna, lava fields from 1536-1669 AD are contained in the data cube.

\subsection{Data}

The Hyperion HSI dataset was acquired on 9 July 2007, with low cloud coverage and a $\pm 5^{\circ}$ looking angle to avoid geometrical distortions. The retrieved Level $1 \mathrm{~T}$ product was radiometrically and geometrically corrected, and also used for spectroscopy studies of Etna [13]. Extensive preprocessing was applied in order to obtain the optimum ground reflectance dataset and ultimately 140 spectral bands were retained, with central wavelengths at $477.7-884.7 \mathrm{~nm}, 973-1336.2 \mathrm{~nm}, 1477.4-$ $1790.2 \mathrm{~nm}$ and 2062.5-2355.2 nm. Preprocessing included: (i) atmospheric correction; (ii) dimensionality and noise reduction via minimum noise fraction analysis; (iii) vegetation masking using the Normalized Difference Vegetation Index (NDVI) values; (iv) active area segregation: Valle del Bove intense current eruptive activity was masked from the initial image; and lastly (v) formation masking: digitization of the MF1 formation within the sensor's FOV, using the formation's boundaries as depicted in the geological map.

\subsection{Endmember Extraction}

Endmembers were retrieved through regions of interest (ROIs) that were strictly constrained within denser lava deposition fields and close to the eruption vents. Borderline regions with potential vegetation growth were excluded and ROIs with limited pixels were merged. In addition, spectral profile examination ensured minimum variability within a certain ROI. Since the broader region of the volcano contains small urban and semi-urban areas, ROIs were also selected for these classes. We extracted ROI pixel values manually and retained the spectral information within $25-75 \%$ of the normal distribution of the reflectance values per ROI and per spectral band. Therefore, outliers and non-characteristic signatures were omitted. We then calculated the mean value per band for the total number of pixels, thus defining the associated endmember vector. We retrieved, in total, 13 endmembers corresponding to eight LFs $(1536,1537,1566,1610,1614-24,1634-36,1646-47$ \& 1669 AD), two scoria cones (1610 \& 1646-47 AD) and three artificial materials (industrial, semi-urban and tile rooftop areas).

\subsection{Methods and Adopted Transformations}

In this section, we will describe in detail the adopted signal transformations and unmixing models implemented for the LF segregation. The implicit fractional abundances were very often constrained to be non-negative (NNC) and to sum-to-one (STOC) [14].

\subsubsection{Method 1: Linear Least Squares Unmixing (LLSU) on the Original Image}

This method is based on the linear mixture model, Equation (1), for the original reflectance dataset, assuming that each endmember covers a defined region inside the pixel area and multiple scattering is negligible [14].

$$
\mathbf{y}=\mathbf{M} \boldsymbol{\alpha}+\mathbf{n}
$$

where $y$ is the spectral signature column vector of a certain pixel; $M$ is the matrix containing the $m_{i}, i$ $=1, \ldots, \mathrm{K}$, endmembers in its columns; $\alpha$ is the abundance vector; and $n$ is the additive white noise. As for the unmixing method, we used a linear least squares non-negative approach, where the estimated abundances [15], derived from a suitable defined Lagrangian function, are given by Equation (2): 


$$
\boldsymbol{\alpha}_{N C L S}=\left(\mathbf{M}^{\mathrm{T}} \mathbf{M}\right)^{-1} \mathbf{M}^{\mathrm{T}} \mathbf{y}-\left(\mathbf{M}^{\mathrm{T}} \mathbf{M}\right)^{-\mathbf{1}} \lambda
$$

\subsubsection{Method 2: LLSU on the Reduced Channel Domain}

Since the removal of multi-collinearity improves the performance of spectral unmixing, and based on the assumption that LFs of the same eruptive cycle can be distinguished by the dataset's intrinsic dimensionality, we removed the redundant spectral information. For this a feature selection (FS) code was used that exploits the sparse unmixing Bayesian inference Iterative Conditional Expectations (BI-ICE) algorithm [16]. Consequently, the total number of endmembers was defined considering a sparsity promoting scheme, hence the extracted endmember matrix is tall by definition. The output of the FS is a set of estimated vectors, the first of which contains a spectral band index with a decreasing significance order, while the second contains the ranks denoting the level of significance of each of these bands. We retained only the first 22 bands with higher significance, reduced the initial image dimension accordingly and then again performed an LLSU scheme.

\subsubsection{Method 3: LLSU with Fast Fourier Transform (FFT) on Selected Frequencies}

For the implementation of this method, the FFT was performed on each of the pixel vectors in the original dataset and on each signature vector in the endmember matrix. Again, the adopted transformation was integrated into an LLSU model. In this case, we implemented the real number of input arguments as a linear combination of the squared imaginary and real parts of the image/endmember matrices. Subsequently, the image was dimensionally reduced in a subspace of 20 frequencies in the frequency domain, which preserved the total energy content. The advantage of this approach is that it counteracts the computationally intensive and time-costly FS.

\subsubsection{Method 4: Bilinear Unmixing (BLU) with the Enhanced Endmember Matrix}

Due to the complex structure of the deposited volcanic deposits, nonlinear effects still exist at macroscopic scales. Therefore, the linear representation of mixed pixels lacks the required detail between secondary light interactions with the on-site materials. In this paper, the nonlinear representation of mixed ground components is inserted through their correlation within the pixel area and the implementation of the interactions in a bilinear mixture model (Equation (3)) [17].

$$
\mathbf{y}=\mathbf{M} \boldsymbol{a}+\sum_{\mathrm{i}=1}^{\mathrm{K}-1} \sum_{\mathrm{j}=\mathrm{i}+1}^{\mathrm{K}} a_{i, j} \mathbf{m}_{\mathbf{i}} \odot \mathbf{m}_{\mathbf{j}}+\mathbf{n} \Leftrightarrow \mathbf{y}=\sum_{\mathrm{k}=1}^{K^{*}} a_{k}{ }^{*} \mathbf{m}_{\mathbf{k}}{ }^{*}+\mathbf{n}
$$

where the vector $\mathbf{m}_{\mathbf{i}} \odot \mathbf{m}_{\mathbf{j}}$ results from the elementwise product of $\mathrm{m}_{\mathrm{i}}, \mathrm{m}_{\mathrm{j}}, \mathrm{K}^{*}=1 / 2 \mathrm{~K}(\mathrm{~K}+1)$, constrained for $\alpha_{\mathrm{k}}^{*} \geq 0$. The bilinear approach was applied to the reduced image resulting as in the context of method 2 and was implemented in the LLSU scheme, where we inserted the non-linearity with an enhanced matrix, containing the dot product specified above.

\section{Results}

The tested unmixing methods produced distinct abundance maps for each endmember that were evaluated in terms of: (i) image reconstruction efficiency; and (ii) method optimality.

\subsection{Endmember Abundances}

We indicatively present the abundances of the 1614-24 LF, 1646-47 scoria, industrial areas (Figure 1) and the SSIM index values for each method (Figure 2). The SSIM index is the quantitative evaluation of our results and indicates the degree of agreement between the initial image and the reconstructed image with the estimated abundance vectors. Each of the SSIM plots exhibits different micro-trends, while there are methods that under strict comparison produce similar results. 

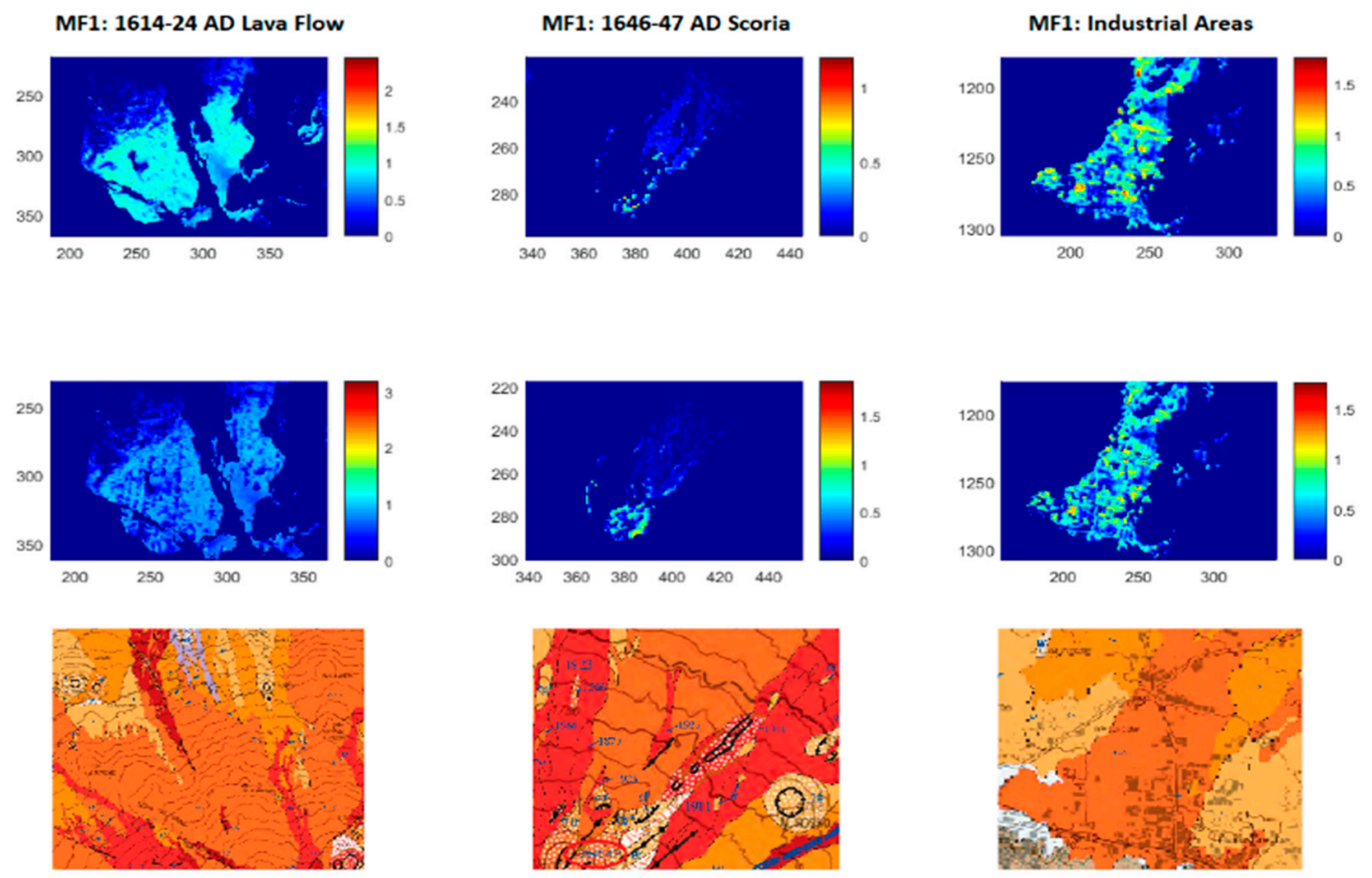

(a)
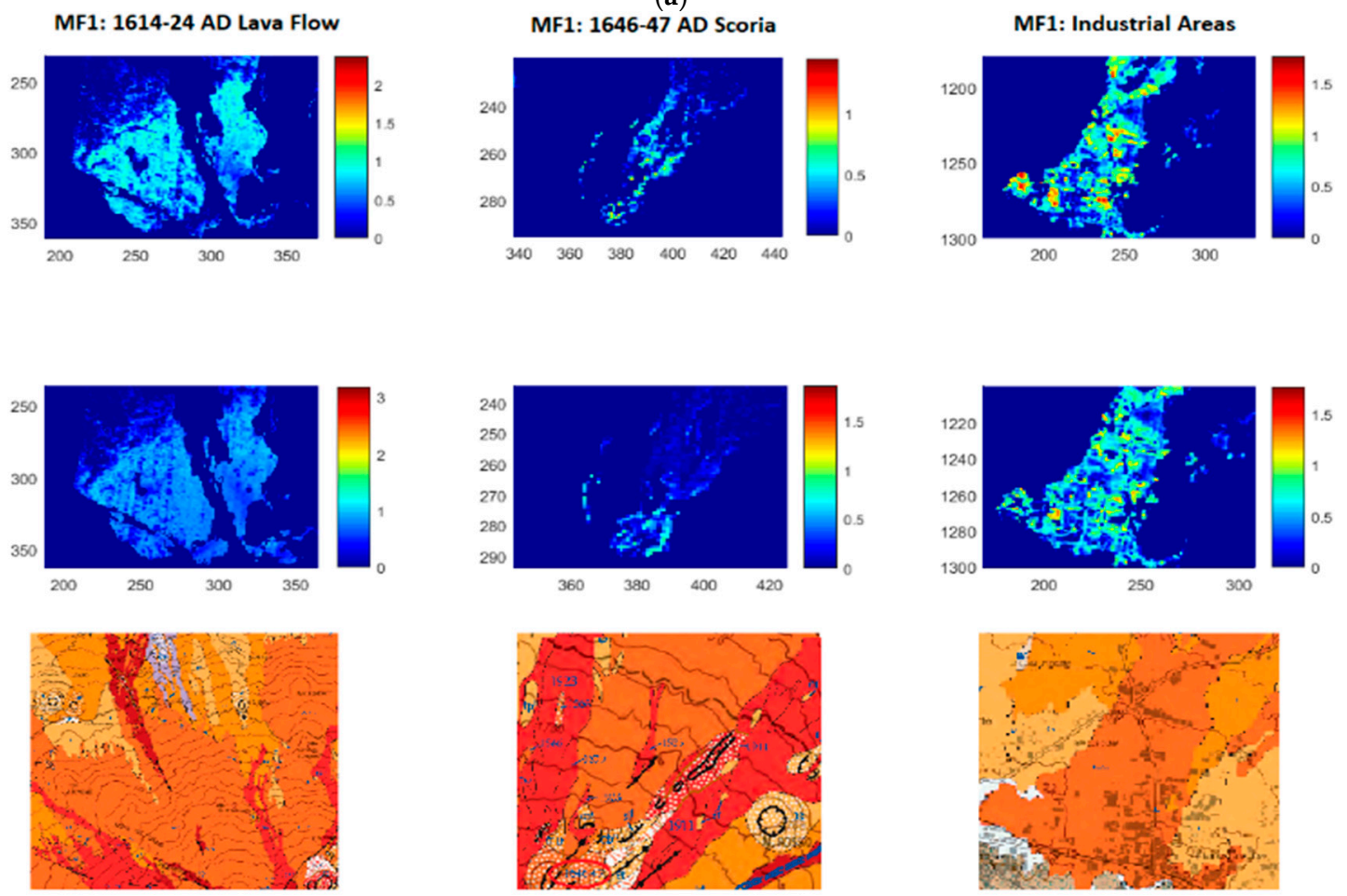

(b)

Figure 1. Abundance maps resulting from the linear and bilinear methods. (a) Top and middle row: Abundances originating from methods 1 and 2; (b) Top and middle row: Abundances originating from methods 3 and 4 . Lower row: the respective region in the geological map. MF1: dark orange areas, MF2: orange and MF3: red areas. 

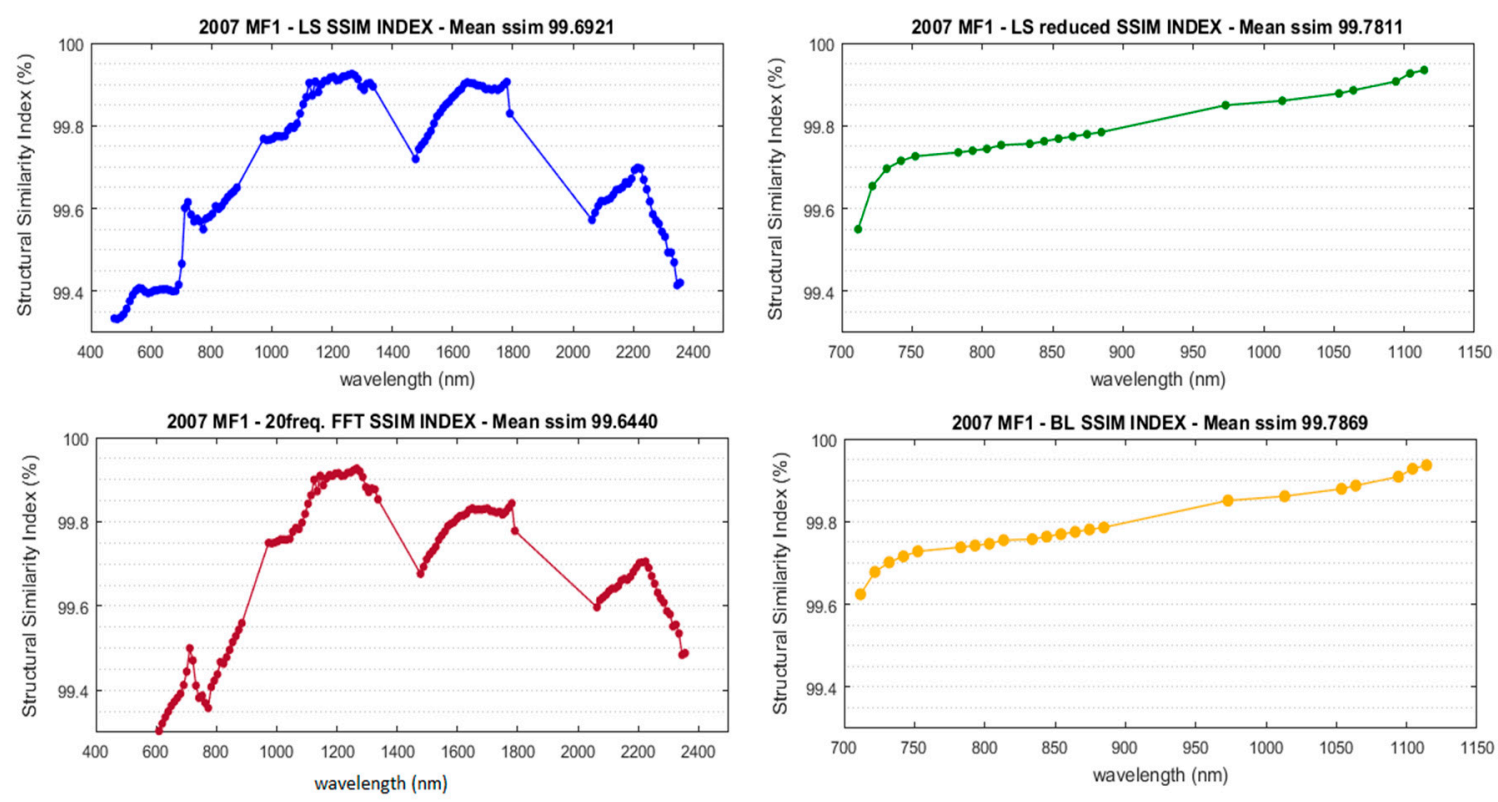

Figure 2. Structural Similarity Index (SSIM) calculated values for methods 1-4 from top left to bottom right. Methods 1 and 3 are on the entire channel domain and produce similar results, while methods 2 and 4 are constrained in the reduced channel domain. Linear interpolation is performed between the resulting values and sharp changes are attributed to the lack of intermediate wavelengths due to pre-processing.

\section{Method Efficiency}

Each method was timed during code performance with a simplistic tic-toc Matlab approach. Table 1 provides the optimum method in terms of time efficiency and dimensionality reduction possibilities. To be statistically accurate, the process was recorded five times per method, then the average time duration was calculated along with the respective error. As inferred from the results, the unmixing of the FFT transformed image is the quickest approach, and also had the lowest standard deviation. This was expected considering the fast implementation of the Fourier transform.

Table 1. Timed operation of the unmixing code.

\begin{tabular}{cccc}
\hline \multirow{2}{*}{ METHOD } & $\begin{array}{c}\text { Elapsed Time (s) } \\
\text { MEAN }\end{array}$ & STDV & EFFICIENCY \\
\hline LLSU & 15.094 & \pm 0.235 & - \\
LLSU reduced & 12.497 & \pm 0.161 & Time \\
FFT 20Freq. & 12.342 & \pm 0.149 & Time \& Dimensionality Reduction \\
BLSU & 32.919 & \pm 0.343 & - \\
\hline
\end{tabular}

\section{Discussion}

The evaluation of the resulting abundance maps, per endmember, carried out via a two-stage process: (a) tracing volcanic product abundances and inter-correlation on a per-method comparison to ground truth; and (b) delimiting lava flows using different methods to extract comparative results, given the reconstruction efficiency for each method. Since the geological map was primarily used for the demarcation of the MF1 formation, we did not provide a definite LF mapping, but rather a spatial verification of each product's presence on the MF1. The above results show that the majority of LFs were accurately delineated by both the linear- and bilinear-based unmixing approches. As expected, higher abundance values were consistently attributed to the ROI areas over the different lava fields. Areas with man-made material were also clearly distinguished. Minor confusions were observed, mainly for products with a similar mineralogical composition and spectral features, such as the scoria cones. Specifically, the observed low abundances in the same areas for different methods can be 
attributed to the lack of low frequency information due to the dimensionality reduction of the reduced datasets. Generally, all adopted methods showed a reconstruction efficiency greater than 99.5\%, denoting the successful implementation of unmixing. Each of the SSIMs exhibited different micro-trends, while there were methods that under strict comparison produced the same reconstruction results. In terms of method effciency, the LLSU and LLSU with FFT for the selected frequencies exhibited the same SSIM trends with respect to the wavelength content and slightly varying SSIM mean values.

\section{Conclusions}

A quantitative analysis of the application of potential spectral unmixing methods on Etnian Lava fields was presented. We concluded that the selection of appropriate regions of interest in such a diverse volcanic environment, considering the particularities of the Hyperion dataset, is of great importance in order to obtain accurate unmixing results. Considering the basic assumptions of the LLSU with fast Fourier transform (FFT) on the selected frequencies method and the subsequent reconstruction degree, we concluded that this method obtains efficient dimensionality reduction of the initial abundance spectral information, with high unmixing accuracy. To the best of our knowledge, the volcanic products of Etna are studied mostly from field measurements, complemented by satellite images, and there are no references to the application of hyperspectral unmixing techniques to Etnian lava fields. Hence, the present research is innovative since signal processing approaches were tailored to a multi-diverse volcanic environment.

$\begin{array}{ll}\text { Abbreviations } \\ \text { HIS } & \text { Hyperspectral Imaging } \\ \text { LF } & \text { Lava Flow } \\ \text { LLSU } & \text { Linear Least Squares Unmixing } \\ \text { BLU } & \text { Bilinear Unmixing } \\ \text { SU } & \text { Spectra Unmixing } \\ \text { FFT } & \text { Fast Fourier Transform } \\ \text { SSIM } & \text { Structural Similarity Index } \\ \text { FS } & \text { Feature Selection }\end{array}$

\section{References}

1. Keshava, N.; Mustard, J.F. Spectral Unmixing. IEEE SPM 2002, 19, 44-57, doi: 10.1109/79.974727

2. Foody, G.M. Sub-Pixel Methods in Remote Sensing. In Remote Sensing Image Analysis: Including the Spatial Domain; Springer: Dordrecht, The Netherlands, 2004; doi:10.1007/978-1-4020-2560-03.

3. Dobigeon, N.; Altmann, Y.; Brun, N.; Moussaoui, S. Linear and Nonlinear Unmixing in Hyperspectral Imaging. Data Handl. Sci. Technol. Elsevier 2016, 30, 185-224.

4. Wang, L.; Zhao, C. Hyperspectral Image Processing; Springer: Berlin/Heidelberg, Germany, 2016; doi:10.1007/978-3-662-47456-3.

5. Singh, K.D.; Ramakrishnan, D. A Comparative Study of signal transformation techniques in automated spectral unmixing of infrared spectra for remote sensing applications. IJRS 2017, 38, 1235-1257.

6. Lentini, F. The Geology of the Mt. Etna Basement. In Mount Etna Volcano, a Review of Recent Earth Sciences Studies; Romano, R., Ed.; Memorie Della Societa Geologica Italiana:, Roma, Italy, 1982; Volume 23, pp. 7 25.

7. Branca, S.; Coltelli, M.; Groppelli, G. Geological map of Etna volcano, 1:50,000 scale. Ital. J. Geosci. 2011, 130, 265-291, doi: 10.3301/IJG.2011.15

8. Allard, P.; Behncke, B.; D'Amico, S.; Neri, M.; Gambino, S. Mount Etna 1993-2005: Anatomy of an evolving eruptive cycle. Elsevier Earth-Sci. Rev. 2006, 78, 85-114., doi: 10.1016/j.earscirev.2006.04.002

9. Spinetti, C.; Neri, M.; Salvatori, R.; Buongiorno, M.F. Spectral properties of volcanic materials from hyperspectral field and satellite data compared with LiDAR data at Mt. Etna. IJAEOG 2009, 11, 142-155, doi: 10.4236/ars.2014.34016

10. Sgavetti, M.; Pompilio, L.; Meli, S. Reflectance spectroscopy (0.3-2.5 $\mu \mathrm{m})$ at Various Vcales for Bulk-rock Identification. Geosphere 2006, 2, 142-160, doi:10.1130/GES00039.1 
11. Zhou, W.; Bovik, A.C.; Sheikh, H.R.; Simoncelli, E.P. Image Quality Assessment: From Error Visibility to Structural Similarity. IEEE TIP 2004, 13, 600-612, doi: 10.1109/TIP.2003.819861

12. Buongiorno, M.F.; Amici, S.; Colini, L.; Di Stefano, G.; Doumaz, F.; Lombardo, V.; Musacchio, M.; Merucci, L.; Pannaccione Apa, M.; Spinetti, C.; et al. ETNA 2003 Field Campaign: Calibration and Validation of Spaceborne and Airborne Instruments for Volcanic Applications; Agenzia Spaziale Italiana Project Report: I/R/157/02, $\mathrm{I} / \mathrm{R} / 203 / 02$.

13. Amici, S.; Piscini, A.; Neri, M. Reflectance Spectra Measurements of Mt. Etna: A Comparison with Multispectral/Hyperspectral Satellite. Sci. Res. 2014, 3, 235-240, doi: 10.4236/ars.2014.34016

14. Heinz, D.C.; Chang, C.I.; Althous, M. Fully Constrained Least-Squares Based Linear Unmixing. In Proceedings of the IEEE International Geoscience and Remote Sensing Symposium, Hamburg, Germany, 28 June-2 July 1999; doi:10.1109/IGARSS.1999.774644

15. Heinz, D.C.; Chang, C.I. Fully Constrained Least Squares Linear Spectral Mixture Analysis Method for Material Quantification in Hyperspectral Imagery. IEEE TGRS 2001, 39, 529-545, doi: 10.1109/36.911111

16. Themelis, K.; Koutroumbas, K.; Rontogiannis, A. A Novel Hierarchical Bayesian Approach for Sparse Semisupervised Hyperspectral Unmixing. IEEE TSP 2012, 60, 585-599, doi: 10.1109/TSP.2011.2174052

17. Altmann, Y.; Dobigeon, N.; Tourneret, J.Y. Bilinear Models for Nonlinear Unmixing of Hyperspectral Images. In Proceedings of the 3rd Workshop on Hyperspectral Image and Signal Processing: Evolution in Remote Sensing (WHISPERS), Lisbon, Portugal, 6-9 June 2011; doi: 10.1109/WHISPERS.2011.6080928

(C) 2018 by the authors. Licensee MDPI, Basel, Switzerland. This article is an open access article distributed under the terms and conditions of the Creative Commons Attribution (CC BY) license (http://creativecommons.org/licenses/by/4.0/). 